\title{
The Shortest Confidence Interval for the Mean of a Normal Distribution
}

\author{
Traoré Boubakar ${ }^{1}$, Diabaté Lassina ${ }^{1}$, Touré Belco ${ }^{2} \&$ Fané Abdou ${ }^{2}$ \\ ${ }^{1}$ Faculté des Sciences Economiques et de Gestion (F.S.E.G), Bamako-Mali \\ ${ }^{2}$ Faculté des Sciences et Techniques (F.S.T), Bamako-Mali \\ Correspondence: Diabaté Lassina, Faculté des Sciences Economiques et de Gestion (F.S.E.G), Bamako-Mali. \\ E-mail: fseggroupe@gmail.com
}

Received: November 30, 2017

Accepted: December 20, 2017 Online Published: January 18, 2018

doi:10.5539/ijsp.v7n2p33

URL: https://doi.org/10.5539/ijsp.v7n2p33

\begin{abstract}
An interesting topic in mathematical statistics is that of the construction of the confidence intervals. Two kinds of intervals which are both based on the method of pivotal quantity are the shortest confidence interval and the equal tail confidence intervals. The aim of this paper is to clarify and comment on the finding of such intervals and to investigation the relation between the two kinds of intervals. In particular, we will give a construction technique of the shortest confidence intervals for the mean of the standard normal distribution. Examples illustrating the use of this technique are given.
\end{abstract}

Keywords: estimation, point estimation, estimation interval, shortest length, unimodal

2010 Mathematics Subject Classifications: 62Exx; 62Fxx; 62Qxx; 93E10.

\section{Introduction}

Let $X$ be a real value random variable from the density $f(x ; \rho)$ and consider the parameter $\rho$ as a fixed unknown quantity. If we seek and interval for $\rho$, then it is well known that the standard method for obtaining confidence intervals for $\rho$ is the pivotal quantity method. (cf. Huzurbazar (1955), Guenther (1969, 1987), Dahiya and Guttman (1982), Ferentinos (1987, 1988, 1990), Juola (1993), Ferentinos and Kourouklis (1990), Kirmani (1990), Cesalla and Berger (2002), Rohatgi and Saleh (2001)...e.t.c).

Let $Z\left(X_{1}, X_{2}, \ldots, X_{n} ; \rho\right)$ be a pivotal quantity where $X_{1}, X_{2}, \ldots, X_{n}$ is a random from the distribution of $f(x ; \rho)$. The probability statement

$$
P_{\rho}\left(z_{1} \leq Z \leq z_{2}\right)=1-\alpha
$$

is converted (when possible) to

$$
P_{\rho}\left(z^{*}{ }_{1} \leq \rho \leq z^{*}{ }_{2}\right)=1-\alpha
$$

If constants $z_{1}, z_{2}$ in (1.1) can be found so that $\left(z^{*}{ }_{2}-z^{*}{ }_{1}\right)$ is minimum, then the interval $\left[z^{*}{ }_{1}, z^{*}{ }_{2}\right]$ is said to be the shortest confidence interval based on $Z$. On the other hand if constants $z_{1}, z_{2}$ in (1.1) can be determined so that

$$
P_{\rho}\left(Z<z_{1}\right)=\frac{\alpha}{2} \quad \text { and } \quad P_{\rho}\left(Z>z_{2}\right)=\frac{\alpha}{2}
$$

then the interval $\left[Z^{*}{ }_{1}, Z^{*}{ }_{2}\right]$ is said to be an equal tails confidence interval.

The aim of this work is to clarify and comment on problems that emerge at the process of finding, to investigate the relation of equality of length of these. In particular, we will give a construction technique of the shortest confidence intervals for the mean of the standard normal distribution.

\section{Method for Evaluating Interval Estimators}

Generally, we want to have confidence intervals with high confidence coefficients as well as small size/length.

Problem is: for a given confidence coefficient $(1-\alpha)$ find the confidence interval with the shortest length.

Let $X$ be a random variable such that $X \sim N\left(\mu=E(X), \sigma^{2}\right)$ (the normal distribution) and $X_{1}, X_{2}, \ldots, X_{n}$ a random $n-$ sample of $X$ with $\sigma^{2}$ known. We then know that a good point estimator of $\mu$ is $\bar{X}$.

As derived above, $\bar{X}=\frac{X_{1}+X_{2}+\cdots+X_{n}}{n} \sim N\left(\mu, \frac{\sigma}{\sqrt{n}}\right)$ and $Z(\bar{X}, \mu)=\frac{\bar{X}-\mu}{\frac{\sigma}{\sqrt{n}}} \sim N(0,1)$ is pivotal, therefore any $[a ; b]$ satisfying 


$$
P_{\mu}(\mathrm{a} \leq \mathrm{Z} \leq \mathrm{b})=\Phi(\mathrm{b})-\Phi(\mathrm{a})=1-\alpha
$$

Yields a corresponding $(1-\alpha)$ confidence interval for the mean $\mu$ :

$$
\left\{\mu: \bar{X}-b \frac{\sigma}{\sqrt{n}} \leq \mu \leq \bar{X}-a \frac{\sigma}{\sqrt{n}}\right\} .
$$

Now we want to choose $[a ; b]$ so that $b-a$ is the shortest length possible for a given confidence coefficient $(1-\alpha)$. It turns out that the symmetric solution $a=-b$ is optimal here. The symmetric solution is

$$
1-\alpha=\Phi(\mathrm{b})-\Phi(\mathrm{a})=\Phi(\mathrm{b})-\Phi(-\mathrm{b})=2 \Phi(\mathrm{b})-1 \Rightarrow b=\Phi^{-1}\left(1-\frac{\alpha}{2}\right) .
$$

This result generalizes to any sampling distribution that is unimodal.

Theorem 1: Let $f$ be a unimodal probability density function. If interval $[a ; b]$ satisfies:

$$
\int_{a}^{b} f(x) d x=1-\alpha
$$

$$
f(a)=f(b)>0
$$

(iii) $\quad a \leq x^{*} \leq b$ where $x^{*}$ is the mode of $f$,

then $[a ; b]$ is the shortest of all intervals that satisfy $(i)$.

Theorem 2: Let $\theta$ and $T(X)$ be real-valued.

Let $U(X)$ be a positive statistic. Suppose that $\frac{T(X)-\theta}{U(X)}$ is a pivotal having a probability density function $f$ that is unimodal at $x_{0} \in \mathbb{R}$. Consider the following class of confidence intervals for $\theta$ :

$$
C=\left\{[T-b U, T-a U]: b \in \mathbb{R}, a \in \mathbb{R}, \int_{a}^{b} f(x) d x=1-\alpha\right\}
$$

If $\left[T-b_{*} U, T-a_{*} U\right] \in C ; f\left(b_{*}\right)=f\left(a_{*}\right)>0$, and $a_{*} \leq x_{0} \leq b_{*}$ then the interval

$\left[T-b_{*} U, T-a_{*} U\right]$ has the shortest length within $C$.

Proof: see references.

\section{Results}

Suppose throughout this part: Let $X$ be a random variable such that $X \sim N\left(\mu=E(X), \sigma^{2}\right)$ (the normal distribution) and $X_{1}, X_{2}, \ldots, X_{n}$ a random $n$ - sample of $X$ with $\sigma^{2}$ known. We then know that a good point estimator of $\mu$ is $\bar{X}$.

Problem is: choose $[a ; b]$ so that $L(a, b)=b-a$ is the shortest length possible for a given confidence coefficient $(1-\alpha)$.

\subsection{Calculation of $a$ and $b$}

The following result provides a general method of finding confidence intervals and covers most cases in practice.

Theorem 3: Let $f$ be a unimodal probability density function of the standard normal distribution. If the reals a and $b$ satisfies:

$$
\left\{\begin{array}{c}
\operatorname{Min}(b-a) \\
\text { subject to }: \int_{a}^{b} f(z) d z=1-\alpha
\end{array}\right.
$$

Then $\left[\bar{X}-b \frac{\sigma}{\sqrt{n}} ; \bar{X}-a \frac{\sigma}{\sqrt{n}}\right] \quad$ is the shortest confidence interval for the mean $\mu$.

Proof:

See Theorem 1: $[\mathrm{a}, \mathrm{b}]$ is the shortest of all intervals that satisfy (i) so that $L(a, b)=b-a$ is the shortest length possible for a given confidence coefficient $(1-\alpha)$. Therefore the confidence interval for the mean is $\left[\bar{X}-b \frac{\sigma}{\sqrt{n}} ; \bar{X}-\right.$ 
$\left.a \frac{\sigma}{\sqrt{n}}\right]$. The length of this confidence interval at level $1-\alpha$ is $K=(b-a) \frac{\sigma}{\sqrt{n}}$.

To find the shortest confidence interval for the mean at level $1-\alpha$ is to minimize the length $L(a, b)=b-a$ subject to: $\int_{a}^{b} f(z) d z=1-\alpha$.

Theorem 4: Let $f$ be a unimodal probability density function of the standard normal distribution. If $\left[\bar{X}-b \frac{\sigma}{\sqrt{n}} ; \bar{X}-a \frac{\sigma}{\sqrt{n}}\right]$ is the shortest confidence interval for the mean $\mu$, then $b=\sqrt{-2 \ln (\lambda \sqrt{2 \pi})}$ and $a=$ $-\sqrt{-2 \ln (\lambda \sqrt{2 \pi})}$ with $\lambda \in] 0, \frac{1}{\sqrt{2 \pi}}[$.

\section{Proof:}

From (i) and (ii), we have: $\left\{\begin{array}{c}f(a)=f(b) \\ \int_{a}^{b} f(z) d z=1-\alpha\end{array}\right.$

$f(a)=f(b) \Leftrightarrow a=b$ or $a=-b$ with $a \neq b$ (to reject $a=b$ ). Therefore $a=-b$.

Finding $a$ and $b$ amounts to solving the equation $f(z)=\lambda$ that is to say $\int_{a}^{b}(f(x) d x-\lambda)=1-\alpha$.

After studying the function $f(z)$, we find the table of variation of $f(z)$ :

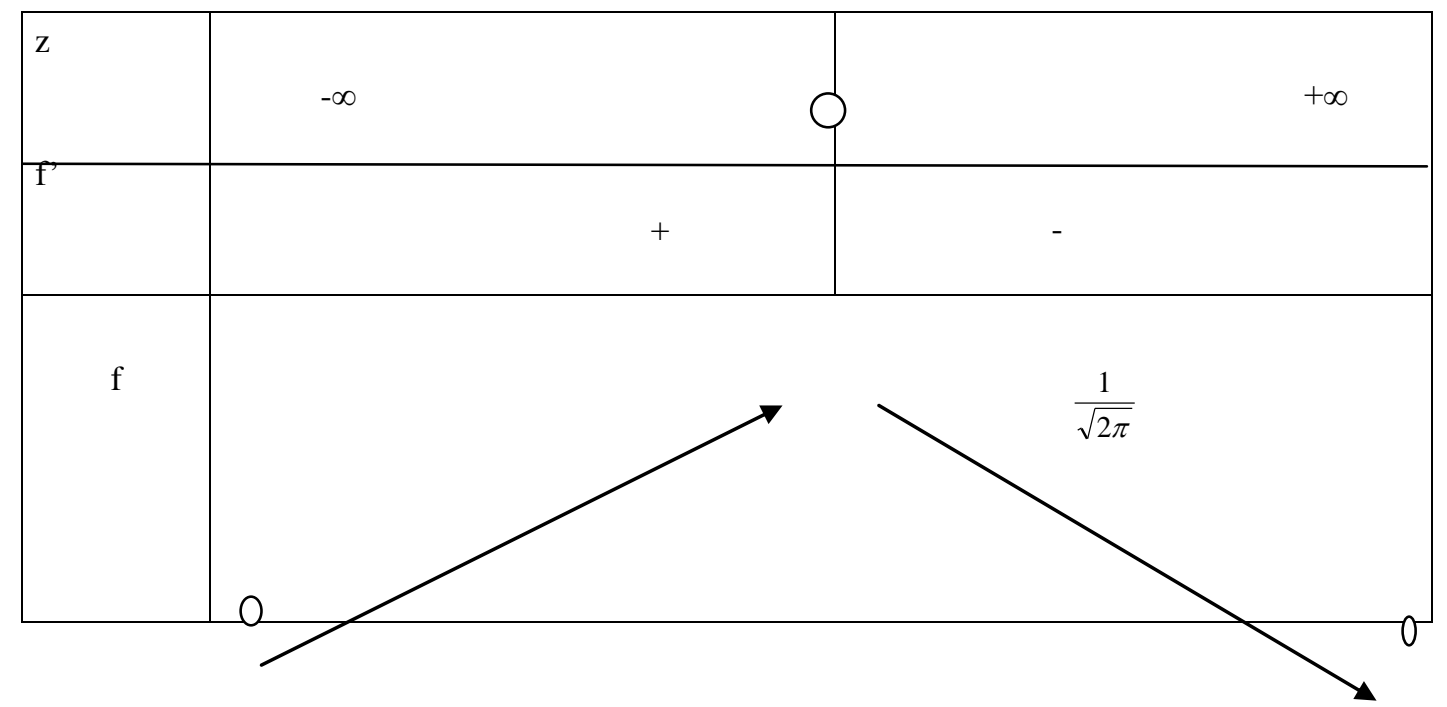




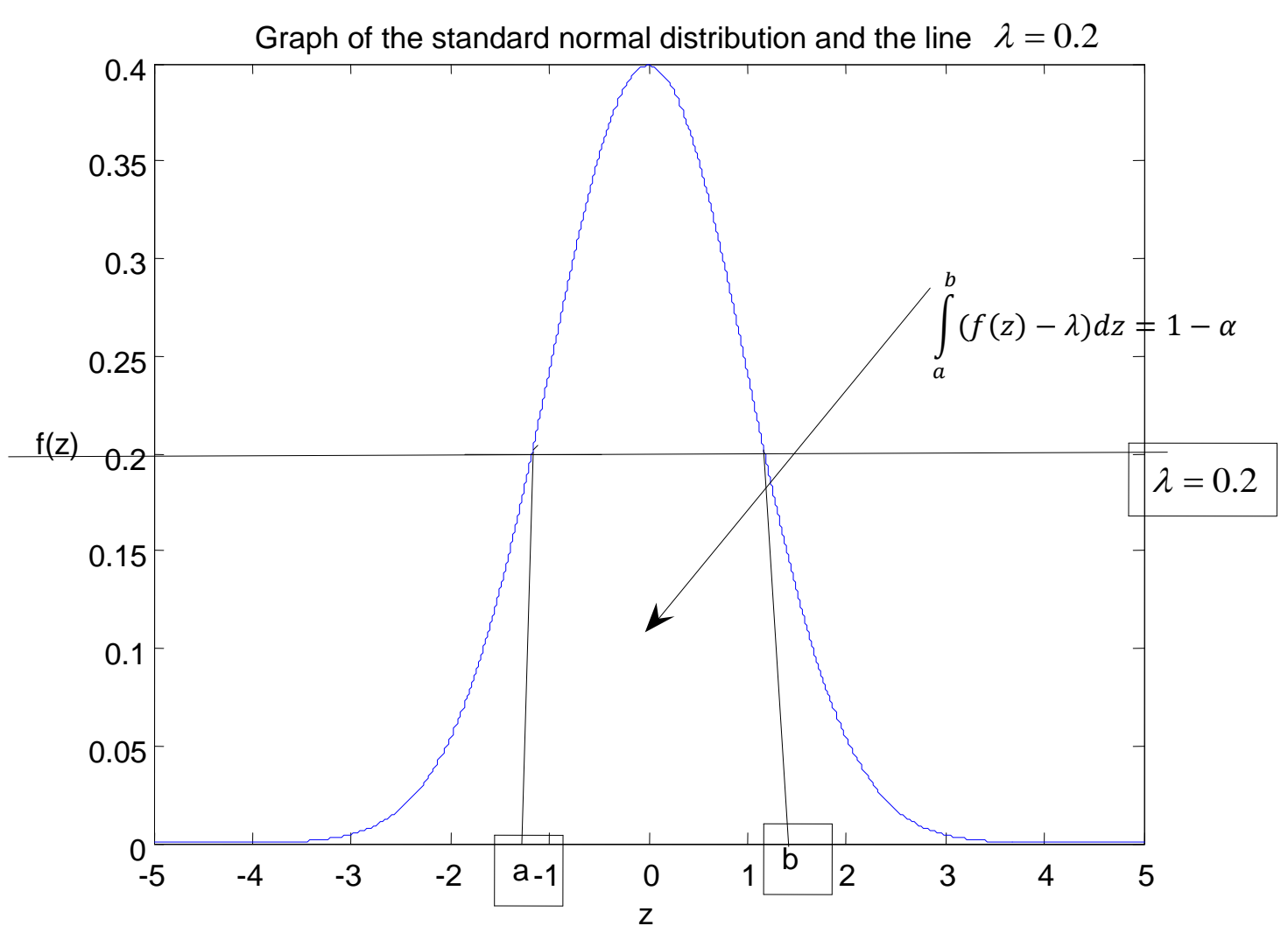

Solve $f(z)=\lambda$ for $\lambda \in] 0, \frac{1}{\sqrt{2 \pi}}\left[: \frac{1}{\sqrt{2 \pi}} e^{\frac{-z^{2}}{2}}=\lambda \Rightarrow z= \pm \sqrt{-2 \ln (\lambda \sqrt{2 \pi})}\right.$.

Therefore Then $b=\sqrt{-2 \ln (\lambda \sqrt{2 \pi})}$ and $a=-\sqrt{-2 \ln (\lambda \sqrt{2 \pi})}$ with $\lambda \in] 0, \frac{1}{\sqrt{2 \pi}}[$.

Corollary: Let $f$ be a unimodal probability density function of the standard normal distribution. If $\left[\bar{X}-b \frac{\sigma}{\sqrt{n}} ; \bar{X}-a \frac{\sigma}{\sqrt{n}}\right]$ is the shortest confidence interval for the mean $\mu$, then

(i) $\quad b=\sqrt{-2 \ln (\lambda \sqrt{2 \pi})}$ and $a=-\sqrt{-2 \ln (\lambda \sqrt{2 \pi})}$ with $\lambda \in] 0, \frac{1}{\sqrt{2 \pi}}[$ and

(ii) $\lambda=\alpha x_{1} x_{2} \ldots x_{k}$ for $0,01 \leq \alpha \leq 0,1$ and $x_{i} \in\{0,1,2, \ldots, 9\}, 1 \leq i \leq k$. 


\subsection{Examples}

\begin{tabular}{|c|c|c|c|c|c|c|c|}
\hline$\alpha$ & $\lambda \in] 0, \frac{1}{\sqrt{2 \pi}}[$ & Equal -Tail & Length $L_{1}$ & $\begin{array}{l}\text { Shortest } \\
\text { Confidence } \\
\text { interval }\end{array}$ & Length $L_{2}$ & $\left|L_{1}-L_{2}\right|$ & Relative error \\
\hline 0,01 & $\lambda=0,01446$ & $\begin{array}{c}a=-2,5760 \\
b=2,5760\end{array}$ & 5,1520 & $\begin{array}{l}a=-2,575822 \\
b=2,575822\end{array}$ & 5,151644 & 0,000356 & 0,00006909938 \\
\hline 0,02 & $\lambda=0,026652$ & $\begin{array}{c}a=-2,326 \\
b=2,326\end{array}$ & 4,652 & $\begin{array}{l}a=-2,32635 \\
b=2,32635\end{array}$ & 4,6527 & 0,0007 & 0,0001504729 \\
\hline 0,03 & $\lambda=0,03787$ & $\begin{array}{c}a=-2,170 \\
b=2,170\end{array}$ & 4,340 & $\begin{array}{l}a=-2,170096 \\
b=2,170096\end{array}$ & 4,340192 & 0,000192 & 0,000044239 \\
\hline 0,04 & $\lambda=0,048418$ & $\begin{array}{c}a=-2,054 \\
b=2,054\end{array}$ & 4,108 & $\begin{array}{l}a=-2,05375 \\
b=2,05375\end{array}$ & 4,1075 & 0,0005 & 0,000121713 \\
\hline 0,05 & $\lambda=0,058445$ & $\begin{array}{c}a=-1,9600 \\
b=1,9600\end{array}$ & 3,9200 & $\begin{array}{c}a=-1,959965 \\
b=1,959965\end{array}$ & 3,91993 & 0,00007 & 0,00001785714 \\
\hline 0,06 & $\lambda=0,068042$ & $\begin{array}{c}a=-1,881 \\
b=1,881\end{array}$ & 3,762 & $\begin{array}{l}a=-1,880793 \\
b=1,880793\end{array}$ & 3,761586 & 0,000414 & 0,00011004 \\
\hline 0,07 & $\lambda=0,07727$ & $\begin{array}{c}a=-1,812 \\
b=1,812\end{array}$ & 3,624 & $\begin{array}{l}a=-1,811911 \\
b=1,811911\end{array}$ & 3,623822 & 0,000178 & 0,000049116 \\
\hline 0,08 & $\lambda=0,086174$ & $\begin{array}{c}a=-1,751 \\
b=1,751\end{array}$ & 3,502 & $\begin{array}{l}a=-1,750685 \\
b=1,750685\end{array}$ & 3,50137 & 0,00063 & 0,000179897 \\
\hline 0,09 & $\lambda=0,094787$ & $\begin{array}{c}a=-1,695 \\
b=1,695\end{array}$ & 3,390 & $\begin{array}{l}a=-1,695396 \\
b=1,695396\end{array}$ & 3,390792 & 0,000792 & 0,00023362 \\
\hline 0,1 & $\lambda=0,103136$ & $\begin{array}{c}a=-1,6450 \\
b=1,6450\end{array}$ & 3,2900 & $\begin{array}{c}a=-1,6448 \\
b=1,6448\end{array}$ & 3,2896 & 0,0004 & 0,0001215805 \\
\hline
\end{tabular}




\section{Conclusions}

The technique given in th for is paper for constructing shortest-length confidence intervals is easy to apply. This technique can also be used solving the similar problems. The existence of confidence intervals with the shortest length do not always exist, even when the distribution of the pivotal quantity is symmetric.

\section{References}

Dahiya, R., \& Guttman, I. (1982). Shortest confidence intervals and prediction intervals for log-normal. The Canadian Journal of Statistics, 10(4), 277-291. https://doi.org/10.2307/3556194

Evans, M., \& Shakhatreh, M. (2008). Optimal properties of some Bayesian inferences. Electronic Journal of Statistics, 2 , 1268-1280. https://doi.org/10.1214/07-EJS126

Ferentinos, K. K., \& Karakostas, K. X. More on shortest and equal tails confidence intervals. Department of mathematics - University of Ioannina - Greece.

Ferentions, K. (1987). Shortest confidence intervals and UMVU estimators for families of distributions involving truncation parameters. Metrika, 34, 341-359. https://doi.org/10.1007/BF02613166

Ferentions, K. (1990). Shortest confidence intervals for families of distributions involving truncation parameters. The American Statistician, 44, 167-168.

Ferentions, K., \& Kouroukli, S. (1990). Shortest confidence intervals for families of distributions involving two truncation parameters. Metrika, 37, 353-363. https://doi.org/10.1007/BF02613544

George Casella. Statistical inference: Interval estimation. Sec:9.3, 144.

Guenther, W. C. (1969). Shortest confidence intervals. The American Statistician, 23, 22-25.

Guenther, W. C. (1971). Unbiased confidence intervals. The American Statistician, 25, 51-53.

John, H. M, \& Kurtis, D. F. Numerical methods using matlab. Sec: 7.2, 373-374.

Konstantin, N. N., Nicholas, A. N., \& Edgars, K. V. (2002). Constructing shortest-length confidence intervals. Department of Mathematical Statistics, University of Latvia., 3(1).

Robert, V. H, \& Elliot, A. T. Probability and statistical inference. Chap6, 359-360.

Troendle, J. F., \& Frank, J. (2001). Unbiased confidence intervals for the odds ratio of two independent binomial samples with application to case-control data. Biometrics, 57, 484-489. https://doi.org/10.1111/j.0006-341X.2001.00484.X

Wall, M. M., Boen, J., \& Tweedie, R. (2001). An effective confidence interval for the mean with samples of size one and two. The American Statistician, 55(2), 102-105. https://doi.org/10.1198/000313001750358400

\section{Copyrights}

Copyright for this article is retained by the author(s), with first publication rights granted to the journal.

This is an open-access article distributed under the terms and conditions of the Creative Commons Attribution license (http://creativecommons.org/licenses/by/4.0/). 